$1988,37,1$

удК 517.98

Эве ОЯ

\title{
СВОЙСТВА, НАСЛЕДУЕМЫЕ ПРОСТРАНСТВАМИ С ШАУДЕРОВЫМИ РАЗЛОЖЕНИЯМИ
}

\author{
(Представил Г. Вайникко)
}

1. Пусть $X$ - банахово пространство. Шаудеровым разложением пространства $X$ называется последовательность $\left(p_{k}\right)=\left(p_{k}\right)_{k \geqslant 1}$ непрерывных ненулевых проекторов в $X$ такая, что $p_{k} p_{t}=0$, если $k \neq l$, и $\sum_{k=1}^{\infty} p_{k} x=x$ для всех $x \in X$. Если этот ряд сходится безусловно для любого $x \in X$, то шаудерово разложение $\left(p_{k}\right)$ называется безусловным. Шаудерово разложение $\left(p_{k}\right)$ для $X$ называется ограниченно полным, если каждая ограниченная последовательность $\left(\sum_{k=1}^{n} x_{k}\right)_{n \geqslant 1}$, - где $x_{k} \in \operatorname{Im} p_{k}$, сходится. Если последовательность сопряженных операторов $\left(p_{k}^{*}\right)_{k \geqslant 1}$ является шаудеровым разложением для сопряженного пространства $X^{*}$, то шаудерово разложение $\left(p_{k}\right)$ пространства $X$ называется натягивающим.

Пространственным идеалом $B$ называется всякая совокупность банаховых пространств, которая содержит все одномерные пространства и удовлетворяет следующим условиям:

$1^{\circ}$ если $X \in B$ и $X_{0}$ изоморфно некоторому дополняемому подпространству в $X$, то $X_{0} \in B$;

$2^{\circ}$ если $X_{1}, X_{2} \in B$, то $X_{1} \times X_{2} \in B$, где прямое произведение наделено максимум-нормой.

Пусть $X$ - банахово пространство с шаудеровым разложением $\left(p_{k}\right)$. Во многих работах по существу изучается следующий вопрос: какие свойства пространств $\operatorname{Im} p_{k}, k \in \mathbf{N}$, передаются пространству $X$ ? Другими словами, при каких условиях $X \in B$, где $B-$ заданный пространственный идеал? Из определения пространственного идеала ясно следующее необходимое условие:

$$
X \in B \Rightarrow \operatorname{Im} p_{k} \in B, \quad k \in \mathbf{N} .
$$

В связи с этим, мы можем поставить свой вопрос в следующей форме. Когда имеет место импликация

$$
\operatorname{Im} p_{k} \in B, \quad k \in \mathbf{N} \Rightarrow X \in B \text { ? }
$$

Для шаудерова разложения $\left(p_{k}\right)$ введем обозначение $P_{n}=$ $=\sum_{k=1}^{n} p_{k}, n \in \mathrm{N}$. Так как $\operatorname{Im} P_{n}$ является изоморфным произведению $\operatorname{Im} p_{1} \times \operatorname{Im} p_{2} \times \ldots \times \operatorname{Im} p_{n}$, то

$$
\operatorname{Im} p_{k} \in B, \quad k \in \mathbf{N} \Rightarrow \operatorname{Im} P_{n} \in B, \quad n \in \mathbf{N} .
$$

Это все, что можно сказать в общем случае. Что касается конкретных случаев, то, например, легко проверяется, что импликация (1) верна для идеала сепарабельных пространств. Дж. Дж. М. Шадвиком ${ }^{\left[{ }^{1}\right]}$ 
доказано, что импликация (1) верна для идеала слабо компактно порожденных пространств. А согласно результату Б. Л. Сандерса $\left[{ }^{2}\right]$, для идеала рефлексивных пространств импликация (1) верна, если шаудерово разложение $\left(p_{k}\right)$ ограниченно полное и натягивающее, причем из рефлексивности пространства следуют ограниченная полнота и натягиваемость любого его шаудерова разложения. Автором $\left[{ }^{3}\right]$ показано, что в случае безусловного ограниченно полного шаудерова разложения импликация (1) верна для идеала слабо секвенциально полных пространств, причем любое безусловное шаудерово разложение таких пространств является ограниченно полным.

Мы будем рассматривать следующие классы банаховых пространств:

1) не содержащие подпространств, изоморфных $l_{1}$ (т. е. согласно теореме Розенталя (см., напр., $\left[{ }^{4}\right]$, стр. 99), почти рефлексивные пространства),

2) не содержащие подпространств, изоморфных $c_{0}$ (т. е. пространства со свойством Пелчиньского),

3) не имеющие фактор-пространств, изоморфных $l_{1}$,

4) не имеющие фактор-пространств, изоморфных $c_{0}$.

Все классы 1)-4) являются пространственными идеалами: условие $1^{\circ}$ очевидно, условие $2^{\circ}$ доказано, напр., в [5]. В связи с идеалом 3) отметим, что банахово пространство имеет фактор-пространство, изоморфное $l_{1}$, тогда и только тогда, когда оно содержит дополняемое подпространство, изоморфное $l_{1}$ (см., напр., [ $\left.{ }^{6}\right]$, стр. 57).

Устанавливаются следующие основные результаты, где $X$ - банахово пространство с шаудеровым разложением $\left(p_{k}\right)$.

T е о р е м а 1. Пусть $\operatorname{Im} p_{k}, k \in \mathrm{N}$, не содержит подпространств, изоморфных $l_{1}$. Для того, чтобы $X$ не содержало подпространств, изоморфных $l_{1}$, достаточна, а если $\left(p_{k}\right)$ безусловно, то и необходима натягиваеMOCTb $\left(p_{k}\right)$.

T ео р ема 2. Пусть $\operatorname{Im} p_{k}, k \in \mathrm{N}$, не содержит подпространств,

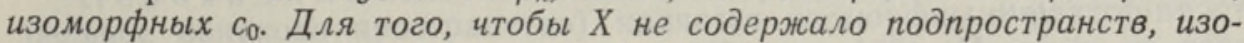
морфных $c_{0}$, достаточна, а если $\left(p_{k}\right)$ безусловно, то и необходима ограниченная полнота $\left(p_{k}\right)$.

Т е ор ем а 3. Пусть $\operatorname{Im} p_{k}, k \in \mathbf{N}$, не имеет фактор-пространств, изоморфных $l_{1}$. Для того, чтобы $X$ не имело фактор-пространств, изоморфных $l_{1}$, достаточна, а если $\left(p_{k}\right)$ безусловно, то и необходима натягиваемость $\left(p_{k}\right)$.

T ео рем а 4. Пусть $\operatorname{Im} p_{k}, k \in \mathrm{N}$, не имеет фактор-пространств, изоморфных $c_{0}$. Для того, чтобы Х не имело фактор-пространств, изоморфных $c_{0}$, достаточна, а если $\left(p_{k}\right)$ безусловно, то и необходима натягиваемость $\left(p_{k}\right) u\left(p_{k}{ }^{*}\right)$.

Теоремы $1-3$ улучшают соответственно теоремы 2,1 и 3 из [ $\left.{ }^{7}\right]$.

2. Необходимость в теоремах 1-3 сразу вытекает из следующего результата [ $\left.{ }^{8}\right]$ (см. доказательства теорем 2, (в), и 1).

Л ем м а 1. Если безусловное шаудерово разложение $\left(p_{k}\right)$ банахова пространства $X$ не является натягивающим (соответственно полным), то $X$ содержит дополняемое подпространство, изоморфное $l_{1}$ (соответственно $c_{0}$ ); более точно, существуют базисная последовательность $\left(u_{i}\right)_{i \geqslant 1}$ в $X$, эквивалентная каноническому базису пространства $l_{1}$ (соответственно $c_{0}$ ), и номера $n_{0}<n_{1}<\ldots$ такие, чTO

$$
u_{i}=\left(P_{n_{t}}-P_{n_{t-1}}\right) u_{i}, \quad i=1,2, \ldots,
$$

и замкнутая линейная оболочка $\left[u_{i}\right]_{i \geqslant 1}$ дополняема в $X$, 
Для доказательства достаточности теорем 1 и 2 нам понадобится следующая лемма, которая доказана в $\left[{ }^{9}\right]$.

Л е м м а 2. Пусть $X-$ - банахово пространство с шаудеровым разложением $\left(p_{k}\right)$ и $Y$ - одно из пространств $c_{0}$ или $l_{1}$. Если X содержит подпространство, изоморфное $Y, a$ Im $\mathrm{p}_{k}, k \in \mathrm{N}$, не содержит подпространств, изоморфных $Y$, то в $X$ существует эквивалентная каноническому базису пространства $Y$ базисная последовательность $\left(u_{i}\right)_{i \geqslant 1}$, удовлетворяющая условию (2).

Достаточность в теоремах 1 и 2 получается последовательным применением леммы 2 и нижеследующей леммы 3.

Л е м м а 3. Пусть $X-$ банахово пространство с шаудеровым разложением $\left(p_{k}\right)$. Если в $X$ существует эквивалентная каноническому базису пространства $l_{1}$ (соответственно $c_{0}$ ) базисная последовательность $\left(u_{i}\right)_{i \geqslant 1}$, удовлетворяющая условию $(2)$, то $\left(p_{k}\right)$ не является натягивающим (соответственно ограниченно полным).

Д ок а з а те льство. Допустим сначала, что $\left(p_{k}\right)$ - натягивающее шаудерово разложение. Пусть $T-$ изоморфизм из $t_{1}$ на $\left[u_{i}\right]_{i \geqslant 1}$ такой, что $T e_{i}=u_{i}, i \in \mathbf{N}$, где $\left(e_{i}\right)_{i \geqslant 1}-$ канонический базис пространства $l_{1}$, и функционал $f \in l_{1}{ }^{*}$ такой, что $f\left(e_{i}\right)=1, i \in \mathbf{N}$. Продолжим $f \circ T^{-1} \in\left[u_{i}\right]_{i \geqslant 1}^{*}$ до функционала $h=\lim _{n} P_{n}{ }^{*} h \in X^{*}$. Тогда $\lim _{i} \| P_{n_{t}}^{*}-$ $\left.-P_{n_{t-1}}^{*}\right) h \|=0$ и, поскольку последовательность $\left(u_{j}\right)_{j \geqslant 1}$ ограничена, то

$$
\lim _{i \rightarrow \infty} \sup _{j \in \mathbf{N}}\left|\left(\left(P_{n_{t}}^{*}-P_{n_{t-1}}^{*}\right) h\right)\left(u_{j}\right)\right|=0 .
$$

С другой стороны, при всех $i \in \mathbf{N}$, ввиду (2), имеем

$$
\sup _{j \in \mathrm{N}}\left|\left(\left(P_{n_{t}}^{*}-P_{n_{t-1}}^{*}\right) h\right)\left(u_{j}\right)\right|=\left|h\left(u_{i}\right)\right|=\left|f\left(e_{i}\right)\right|=1 .
$$

ІІолученное противоречие завершает доказательство первой части леммы.

Допустим теперь, что $\left(p_{k}\right)$ является ограниченно полным. Так как последовательность $\left(u_{i}\right)_{i \geqslant 1}$ эквивалентна каноническому базису пространства $c_{0}$, то последовательность $\left(\sum_{i=1}^{n} u_{i}\right)_{n \geqslant 1}$ ограничена и $u_{i} \nrightarrow 00$. Зведем последовательность $x_{1}=0, \ldots, x_{n_{0}}=0, x_{n_{0}+1}=p_{n_{0}+1} u_{1}, \ldots$, $x_{n_{1}}=p_{n_{1}} u_{1}, x_{n_{1}+1}=p_{n_{1}+1} u_{2}, \ldots, x_{n_{2}}=p_{n_{2}} u_{2}, \ldots$ Тогда $x_{k} \in \operatorname{Im} p_{k}$, $b \in \mathbf{N}$. Поскольку при любом $m>n_{0}$ имеем $m \in\left\{n_{i}+1, \ldots, n_{i+1}\right\}$ (при некотором $i$ ) и, следовательно,

$$
\begin{aligned}
\sum_{k=1}^{m} x_{k} & =\sum_{k=n_{0}+1}^{n_{t}} x_{k}+\sum_{k=n_{t}+1}^{m} x_{k}=\sum_{j=1}^{i} u_{j}+P_{m} u_{i+1}= \\
& =\sum_{j=1}^{i} P_{m} u_{j}+P_{m} u_{i+1}=P_{m}\left(\sum_{j=1}^{i+1} u_{j}\right),
\end{aligned}
$$

то последовательность $\left(\sum_{k=1}^{m} x_{k}\right)_{m \geqslant 1}$ ограничена. В силу ограниченной полноты $\left(p_{k}\right)$ ряд $\sum_{k=1}^{\infty} x_{k}$ сходится, что противоречит условню

$$
\sum_{k=n_{t-1}+1}^{n_{t}} x_{k}=u_{i} \nrightarrow 0
$$

Лемма доказана, 
Для доказательства достаточности теорем 3 и 4 нам понадобится следующая лемма из $\left[{ }^{10}\right]$.

Л емм а 4. Пусть X - банахово пространство с натягивающим шаудеровым разложением $\left(p_{k}\right)$. Если X имеет фактор-пространство, изоморфное $l_{1}$ (соответственно $c_{0}$ ), a Im $p_{k}, k \in \mathbf{N}$, не имеет фактор-пространств, изоморфных $l_{1}$ (соответственно $c_{0}$ ), то существуют базисная последовательность $\left(f_{i}\right)_{i \geqslant 1}$ в $X^{*}$, эквивалентная каноническому базису пространства $c_{0}$ (соответственно $l_{1}$ ), и номера $n_{0}<n_{1}<\ldots$ такие, что

$$
f_{i}=\left(P_{n_{t}}^{*}-P_{n_{t-1}}^{*}\right) f_{i}, \quad i=1,2, \ldots .
$$

3 а м е ч а и е. Если в $X^{*}$, где $X-$ банахово пространство с шаудеровым разложением $\left(p_{k}\right)$, существует эквивалентная каноническому базису пространства $c_{0}$ последовательность $\left(f_{i}\right)_{i \geqslant 1}$, удовлетворяющая условию (3), то, в силу леммы 3 из [ $\left.{ }^{10}\right]$, в $X$ существует эквивалентная каноническому базису пространства $l_{1}$ базисная последовательность $\left(u_{i}\right)_{i \geqslant 1}$, удовлетворяющая равенству $(2)$.

Достаточность в теореме 3 . Пусть $\left(p_{k}\right)$ - натягивающее шаудерово разложение. Допустим, что $X$ имеет фактор-пространство, изоморфное $l_{1}$. Применяя лемму 4 и учитывая замечание к ней, приходим к противоречию с леммой 3 .

Доказ ательство теоремы 4. Достаточность. Пусть $\left(p_{k}\right)$ - натягиваюшее шаудерово разложение, причем шаудерово разложение $\left(p_{k}{ }^{*}\right)$ в $X^{*}$ также натягивающее. Допустим, что $X$ имеет фактор-пространство, изоморфное $c_{0}$, и воспользуемся леммой 4 . Последующее применение леммы 3 к $X^{*}$ с шаудеровым разложением $\left(p_{k}{ }^{*}\right)$ приводит к противоречию с натягиваемостью $\left(p_{k}{ }^{*}\right)$.

Н е обходи мость. Напомним, что $c_{0}$ (и вообще любое сепарабельное банахово пространство) является изоморфным фактор-пространству пространства $l_{1}$ (см., напр., $\left[{ }^{4}\right]$, стр. 108). Поэтому из того, что $X$ и $\operatorname{Im} p_{k}, k \in \mathbf{N}$, не имеют фактор-пространств, изоморфных $c_{0}$, следует, что $X$ и $\operatorname{Im} p_{k}, k \in \mathbf{N}$, не имеют фактор-пространств, изоморфных $l_{1}$. Значит, мы можем воспользоваться теоремой 3 , откуда вытекает натягиваемость $\left(p_{k}\right)$.

В силу натягиваемости $\left(p_{k}\right)$, последовательность $\left(p_{k}{ }^{*}\right)$ представляет собой безусловное шаудерово разложение пространства $X^{*}$. Допустим, что оно не является натягивающим и применим к нему лемму 1. Тем самым мы находим в $X^{*}$ эквивалентную каноническому базису пространства $l_{1}$ базисную последовательность $\left(f_{i}\right)_{i \geqslant 1}$, удовлетворяющую условию (3). К противоречию приводит нас теперь следующий результат, вытекающий из [11] (см. $\left[{ }^{10}\right]$, стр. 90): если $X^{*}$ содержит эквивалентную каноническому базису пространства $l_{1}$ базисную последовательность, сходящуюся к нулю в *-слабой топологии, то $X$ имеет фактор-пространство, изоморфное $c_{0}$. Итак, остается проверить, что $f_{i}(x) \rightarrow 0$ при всех $x \in X$. Для этого достаточно заметить, что (в силу эквивалентности каноническому базису) последовательность $\left(\left\|f_{i}\right\|\right)_{i \geqslant 1}$ ограничена и $f_{i}\left(p_{k} x\right) \rightarrow_{i} 0$ при всех $x \in X, k \in \mathbf{N}$, поскольку для $n_{i-1} \geqslant k$ выполняется равенство

$$
f_{i}\left(p_{k} x\right)=f_{i}\left(\left(P_{n_{t}}-P_{n_{t-1}}\right) p_{k} x\right)=f_{i}\left(\left(\sum_{j=n_{t-1}+1}^{n_{t}} p_{j} \circ p_{k}\right) x\right)=0
$$

Теорема доказана. 
3. Применим теоремы 2 и 4 для быстрого обоснования нижеследующего примера С. Ф. Бэлленота $\left[{ }^{12}\right]$, для обоснования которого в $\left[{ }^{12}\right]$ потребовалось немало технического труда (ср. с доказательством предложения 2.1 в $\left.\left[{ }^{12}\right]\right)$.

Пр и м р. Существует банахово пространство $X$ с безусловным, натягивающим и ограниченно полным шаудеровым разложением $\left(p_{k}\right)$, где $\operatorname{Im} p_{k}, k \in \mathbf{N}$, изоморфно пространству Дюеймса $J$, таким, что шаудерово разложение $\left(p_{k}^{*}\right)$ для $X^{*}$ не является натягивающим.

Пусть $Y=J\left(X_{n}, \varphi_{n}\right)$, где $X_{n}=l_{p}$ с $p=n+1$ и $\varphi_{n}: X_{n} \rightarrow X_{n+1}, n \in \mathbf{N}$, - каноническое вложение. Символ $J\left(X_{n}, \varphi_{n}\right)$ обозначает $J$-сумму пространств $\left(X_{n}\right)_{n \geqslant 1}$, определенную в $\left[{ }^{12}\right]$ и обобщающую естественным образом пространство Джеймса $J$. Принимая во внимание аналогию с $J$, нетрудно показать (см. $\left.\left[{ }^{12}\right]\right)$, что $Y^{*}$ является сепарабельным и $Y^{* *} / Y$ (изометрически) изоморфным $c_{0}$. Последний изоморфизм влечет за собой изоморфизм между $c_{0}^{*}$ и аннулятором $Y^{\perp} \subset Y^{* * *}$. Поскольку $Y^{* * *}=Y^{*} \oplus Y^{\perp}$, то и $Y^{* * *}$ (а значит, также $Y^{* *}$ ) является сепарабельным.

Рассматривая $Y=J\left(X_{n}, \varphi_{n}\right)$ как линейное множество в $X_{1} \times X_{2} \times \ldots$, обозначим при $k \in \mathbf{N}$

$$
\begin{aligned}
& e_{1, k}=\left(e_{k}, 0,0, \ldots\right) \\
& e_{2, k}=\left(0, e_{k}, 0,0, \ldots\right) \\
& e_{3, k}=\left(0,0, e_{k}, 0,0, \ldots\right)
\end{aligned}
$$

где $\left(e_{k}\right)_{k \geqslant 1}-$ канонический базис пространства $l_{p}$. Нетрудно проверить, что последовательность $\left[e_{n, 1}\right]_{n \geqslant 1},\left[e_{n, 2}\right]_{n \geqslant 1}, \ldots$ замкнутых линейных оболочек в $Y$ определяет там безусловное (даже симметричное) шаудерово разложение $\left(q_{k}\right)_{k \geqslant 1}$, где через $q_{k}$ обозначен проектор в $Y$ с $\operatorname{Im} q_{k}=\left[e_{n, k}\right]_{n \geqslant 1}$. Ясно, что $\operatorname{Im} q_{k}, k \in \mathbf{N}$, является (изометрически) изоморфным $J$. Поэтому $\operatorname{Im} q_{k}{ }^{*}$ и $\operatorname{Im} q_{k}{ }^{* *}$ изоморфны соответственно $J^{*}$ и $J^{* * *} ;$ значит, $\operatorname{Im} q_{k}{ }^{* *}$ изоморфно и $J$.

Заметим, что $Y$ и $Y^{*}$ не имеют фактор-пространств, изоморфных $c_{0}$, так как $c_{0}{ }^{* *}$ не сепарабельно и потому не может быть изоморфным фактор-пространству пространств $Y^{* *}$ или $Y^{* * *}$. Вследствие теоремы 4 , $\left(q_{k}{ }^{* *}\right)$ представляет собой натягивающее шаудерово разложение пространства $Y^{* *}$. Оно безусловно, так как $\left(q_{k}\right)$ безусловно. В силу теоремы $2,\left(q_{k}{ }^{* *}\right)$ является также ограниченно полным, поскольку $Y^{* *}$ не содержит подпространств, изоморфных $c_{0}$, так как в противном случае оно, согласно теореме Бессаги-Пелчиньского (см., напр., $\left[{ }^{4}\right]$, стр. $103)$, содержало бы несепарабельное подпространство, изоморфное $l_{\infty}$. Остается обозначить $X=Y^{* *}$ и $p_{k}=q_{k}{ }^{* *}, k \in \mathrm{N}$, и заметить, что согласно теореме $4,\left(p_{k}{ }^{*}\right)$ не может быть натягивающим, поскольку $X$ имеет фактор-пространство, изоморфное $c_{0}$.

Напомним (см. п. 1), что в случае банахова пространства $X$ с шаудеровым разложением $\left(p_{k}\right)$ таким, что $\operatorname{Im} p_{k}, k \in \mathbf{N}$, рефлексивно, $\left(p_{k}\right)$ является натягивающим и ограниченно полным тогда и только тогда, когда $X$ рефлексивно. Значит, из того, что $\operatorname{Im} p_{k}, k \in \mathbf{N}$, рефлексивно, a $\left(p_{k}\right)$ натягивающее и ограниченно полное, следует натягиваемость $\left(p_{k}{ }^{*}\right)$. Вышеприведенный пример показывает, что рефлексивность Im $p_{k}, k \in \mathbf{N}$, является существенным в последнем результате (даже в случае безусловного $\left.\left(p_{k}\right)\right)$, и тем самым представляет собой контрпример теореме 1 из $\left[{ }^{13}\right]$.

Кроме пространства с безусловным шаудеровым разложением, одним из хорошо известных обобщений банахова пространства с без- 
условным базисом является (с точностью до изоморфизма) банахова решетка. Отметим, что не все банаховы теоремы теории банаховых решеток (т. е. теоремы, в формулировках которых используют лишь понятия теории банаховых пространств, но не решеток [ $\left.{ }^{14}\right]$ ) остаются верными в пространствах с безусловным шаудеровым разложением. Так, например, банахова решетка $Z$ не содержит подпространств, изоморфных $l_{1}$ или $c_{0}$, тогда и только тогда, когда $Z^{*}$ не содержит подпространств, изоморфных $l_{1}$ (см., напр., $\left[{ }^{14}\right]$, стр. 166). В то же время, в вышеприведенном примере, согласно теоремам 1 и $2, X$ не содержит подпространств, изоморфных $l_{1}$ или $c_{0}$, а $X^{*}$ содержит подпространство, изоморфное $l_{1}$.

4. В заключение выведем из теорем 1-4 некоторые результаты, связывающие линейно-топологические свойства банахова пространства $X$ с безусловным шаудеровым разложением и его сопряженного пространства $X^{*}$, а также характеризующие рефлексивность $X$. Отметим, что все эти результаты перестают быть верными в классе всех банаховых пространств.

Согласно результату Джонсона-Розенталя (см., напр., [ $\left.{ }^{4}\right]$, стр. 104), сепарабельное банахово пространство имеет фактор-пространство, изоморфное $c_{0}$, если его сопряженное пространство содержит подпространство, изоморфное $l_{1}$. В случае банахова пространства $X$ с безусловным шаудеровым разложением $\left(p_{k}\right)$ имеем следующие два результата.

С л е д с т и е 1. Пусть Im $p_{k}{ }^{*}, k \in \mathbf{N}$, не содержит подпространств, изоморфных $l_{1}$. Если $X^{*}$ имеет фактор-пространство, изоморфное $l_{1}$, то $X$ имеет фактор-пространство, изоморфное $c_{0}$.

Д оказ а тельство. Допустим, что $X$ не имеет фактор-пространств, изоморфных $c_{0}$. Поскольку $\operatorname{Im} p_{k}, k \in \mathbf{N}$, не имеет фактор-пространств, изоморфных $c_{0}$, то по теореме $4\left(p_{k}{ }^{*}\right)$ является натягивающим шаудеровым разложением в $X^{*}$. Применение теоремы 1 приводит к противоречию с тем, что $X^{*}$ содержит подпространство, изоморфное $l_{1}$.

С л е дс т в и е 2. Пусть $\operatorname{Im} p_{k}{ }^{*}, k \in \mathbf{N}$, не имеет фактор-пространств, изоморфных $l_{1}$. Если $X^{*}$ имеет фактор-пространство, изоморфное $l_{1}$, то Х имеет фактор-пространство, изоморфное $c_{0}$.

Д ок а з а т ел ь с т в о. Если существует $k \in \mathbf{N}$ так, что $\operatorname{Im} p_{k}$ имеет фактор-пространство, изоморфное $c_{0}$, то это верно и для $X$. В противном случае рассуждаем, как в предыдущем доказательстве, применяя вместо теоремы 1 теорему 3.

В связи со следствиями 1 и 2 отметим, что, например, $l_{\infty}{ }^{*}$ содержит дополняемое подпространство, изоморфное $l_{1}$ (так как $c_{0}{ }^{*}$ дополняемо в $\left.c_{0}{ }^{* * *}\right)$, однако $l_{\infty}$ не имеет фактор-пространств, изоморфных $c_{0}$ (так как каждое сепарабельное фактор-пространство пространства $l_{\infty}$ рефлексивно (см., напр., $\left[{ }^{6}\right]$, стр. 100, 101)).

Далее, поскольку $c_{0}$ изоморфно фактор-пространству $l_{1}$ (см., напр., $\left[{ }^{4}\right]$, стр. 108), то $l_{\infty}$ содержит подпространство, изоморфное $l_{1}$. Поскольку $l_{\infty}$ не имеет фактор-пространств, изоморфных $l_{1}$, то по теореме Бессаги-Пелчиньского (см., напр., $\left[{ }^{4}\right]$, стр. 103) $l_{\infty}{ }^{*}$ не содержит подпространств, изоморфных $l_{\infty}$. Итак, существует банахово пространство, содержащее подпространство, изоморфное $l_{1}$, сопряженное к которому не содержит подпространств, изоморфных $l_{\infty}$. Однако в случае банахова пространства $X$ с безусловным шаудеровым разложением $\left(p_{k}\right)$ имеем

С л едст в и е 3. Пусть $\operatorname{Im} p_{k}, k \in \mathbf{N}$, не содержит подпространств, изоморфных $l_{1}$. Если $X$ содержит подпространство, изоморфное $l_{1}$, то $X^{*}$ содержит подпространство, изоморфное $l_{\infty}$. 
Д ок а з а тельст в о. Согласно теореме $1,\left(p_{k}\right)$ не является натягивающим. Так как Im $p_{k}, k \in \mathbf{N}$, не имеет и фактор-пространств, изоморфных $l_{1}$, то по теореме 3 у $X$ есть фактор-пространство, изоморфное $l_{1}$. Значит, $X^{*}$ содержит подпространство, изоморфное $l_{\infty}$.

Согласно хорошо известному критерию Джеймса $\left[{ }^{15}\right]$ (см. также [6], стр. 130), банахово пространство $X$, обладающее безусловным базисом, рефлексивно тогда и только тогда, когда ни $X$, ни $X^{*}$ не содержит подпространств, изоморфных $l_{1}$. Г. Я. Лозановский [ $\left.{ }^{16}\right]$ (см. также $\left[{ }^{14}\right]$, стр. 166) заметил, что требование «X не содержит подпространств, изоморфных $l_{1} \gg$ здесь излишне (даже в случае произвольных банаховых решеток). А поскольку сепарабельное банахово пространство имеет фактор-пространство, изоморфное $c_{0}$, тогда и только тогда, когда его сопряженное пространство содержит подпространство, изоморфное $l_{1}$, то условие « $X$ не имеет фактор-пространств, изоморфных $c_{0}$ также равносильно рефлексивности $X$. Следующий результат распространяет эти критерии рефлексивности на банаховы пространства с безусловным шаудеровым разложением.

С л е д с т в и е 4. Пусть $X-$ банахово пространство с безусловным шаудеровым разложением $\left(p_{k}\right)$ таким, что $\operatorname{Im} p_{k}, k \in \mathbf{N}$, рефлексивно. Тогда следующие условия равносильны:

$1^{\circ} X$ рефлексивно,

$2^{\circ} X$ не имеет фактор-пространств, изоморфных $c_{0}$,

$3^{\circ} X^{*}$ не содержит подпространств, изоморфных $l_{1}$.

Д ок а з а тельст в о. Очевидно, $1^{\circ} \Rightarrow 3^{\circ} \Rightarrow 2^{\circ}$. Докажем, что $2^{\circ} \Rightarrow 1^{\circ}$. Пусть выполнено условие $2^{\circ}$. В силу теоремы 4 , шаудерово разложение $\left(p_{k}\right)$ является натягивающим. Далее, по следствию $2 X^{*}$ не имеет фактор-пространств, изоморфных $l_{1}$. Поэтому $X$ не имеет подпространств, изоморфных $c_{0}$, и в силу теоремы $2,\left(p_{k}\right)$ является также ограниченно полным. Рефлексивность $X$ следует теперь из результата Сандерса, упомянутого в п. 1 .

З а м е а н и е. Согласно недавнему результату Е. В. Токарева $\left[{ }^{17}\right]$, условие $2^{\circ}$ в некотором классе банаховых решеток равносильно тому, что всякое сепарабельное фактор-пространство $X$ является рефлексивным.

В связи со следствием 4 отметим, что другие критерии рефлексивности пространства с безусловным шаудеровым разложением, обобщающие и усиливающие известные критерии Джеймса-Дэя-Карлина (см. $\left[{ }^{6}\right]$, стр. 130), можно найти в $\left[{ }^{8}\right]$ и $\left[{ }^{18}\right]$.

Известно, что некоторые критерии рефлексивности банахова пространства с безусловным шаудеровым разложением $\left(p_{k}\right)$ таким, что все Im $p_{k}$ рефлексивны, совпадают с критериями рефлексивности банаховой решетки (см. $\left[{ }^{14}\right]$, стр. 166 ; так, напр., $1^{\circ} \Leftrightarrow 3^{\circ}$ для произвольной банаховой решетки $X$ ), и даже банахова пространства с локальной безусловной структурой (класс которых существенно шире класса банаховых пространств, изоморфных банаховым решеткам) [19]. Поэтому следует отметить, что существует банахово пространство с безусловным шаудеровым разложением $\left(p_{k}\right)$ таким, что $\operatorname{dim}\left(\operatorname{Im} p_{k}\right)=2$, $k \in \mathbf{N}$, которое не имеет локальной безусловной структуры $\left[{ }^{20}\right]$.

\section{ЛИТЕРА Т У Р А}

1. Chadwick, J. J. M. Bull. Austral. Math. Soc., 6, № 1, 133-144 (1972).

2. Sanders, B. L. Proc. Amer. Math. Soc., 16, № 2, 204-208 (1965).

3. Оя Э. Уч. зап. Тартуск. гос. ун-та, № 374, 90-116 (1975).

4. Lindenstrauss, J., Tzafriri, L. Classical Banach Spaces, I. Sequence Spaces. BerlinHeidelberg-New York, Springer Verlag, 1977. 
5. Samuel, C. Math. Scand., 45, № 1, 103-117 (1979).

6. Дэй М. М. Нормированные линейные пространства. М., ИЛ, 1961.

7. Оя Э., Ныльвак А., Олев А., Трахв С. В кн.: Теоретические и прикладные вопросы математики, 1. Тезисы докладов конференции 26-27 сент. 1985 г. Тарту, 1985, 126-128.

8. Оя Э. Уч. зап. Тартуск. гос. ун-та, № 431, $82-97$ (1977).

9. Oja, E. Math. Scand., 51, № 2, 275-288 (1982).

10. Оя Э. Ф. Матем. заметки, 39, № 1, 83-96 (1986).

11. Johnson, W. B., Rosenthal, H. P. Stud. math., 43, № 1, 77-92 (1972).

12. Bellenot, S. F. J. Funct. Anal., 48, № 1, 95-106 (1982).

13. Оя Э. Уч. зап. Тартуск. гос. ун-та, № 374, 117-127 (1975).

14. Бухвалов А. В., Векслер А. И., Лозановский Г. Я. Успехи матем. наук, 34, № 2 (206), 137-183 (1979).

15. James, R. J. Ann. Math., 52, № 3, 518-527 (1950).

16. Лозановский $Г$. Я. Функц. анализ и его прилож., 1, № 3, 92 (1967).

17. Токарев E. B. Сиб. матем. ж., 25, № 2, 205-212 (1984).

18. Оя Э. Уч. зап. Тартуск, гос, ун-та, № 431, 98-103 (1977).

19. Niculescu, C. Rev. roum. math. pures et appl., 23, № 2, 233-242 (1978).

20. Johnson, W. B., Lindenstrauss, J., Schechtman, G. Isr. J. Math., 37, № 1-2, 120-129 (1980).

Тартуский государственный университет

Поступила в редакцию

10/II 1987

Eve OJA

\section{SCHAUDERI LAHUTUSEGA RUUMIDELE PÄRANDUVAD OMADUSED}

On uuritud jadaruumidega $l_{1}$ ja $c_{0}$ isomorfsete alamruumide ja faktorruumide puudumise päranduvust komponentruumidelt Schauderi lahutusega Banachi ruumile. Saadud tulemuste abil on põhjendatud lühidalt S. F. Bellenoti tehniliselt raske näide [ $\left.{ }^{12}\right]$, järeldatud mitmeid tulemusi, mis seovad tingimatu Schauderi lahutusega Banachi ruumi ja tema kaasruumi lineaartopoloogilist struktuuri, ning tuletatud refleksiivsuse kriteeriumid tingimatu Schauderi lahutusega Banachi ruumi jaoks.

\section{PROPERTIES THAT CAN BE INHERITED BY SPACES WITH SCHAUDER DECOMPOSITIONS}

Let $X$ be a Banach space having a Schauder decomposition $\left(p_{k}\right)=\left(p_{k}\right)_{k \geqslant 1}$. Reproducibility properties of the spaces $l_{1}$ and $c_{0}$ in $X$ are studied. The main results are:

The or em 1. Suppose that $\operatorname{Im} p_{k}, k=1,2, \ldots$, contains no subspace isomorphic to $l_{1}$. It is sufficient for $X$ to contain no subspace isomorphic to $l_{1}$, and if $\left(p_{k}\right)$ is unconditional, then it is also necessary that $\left(p_{k}\right)$ is shrinking.

The orem 2. Suppose that $\operatorname{Im} p_{k}, k=1,2, \ldots$, contains no subspace isomorphic to $l_{1}$. It is sufficient for $X$ to have no quotient space isomorphic to $l_{1}$, and if $\left(p_{h}\right)$ is unconditional, then it is also necessary that $\left(p_{k}\right)$ is boundedly complete.

The or em 3. Suppose that $\operatorname{Im} p_{k}, k=1,2, \ldots$, has no quotient space isomorphic to $l_{1}$. It is sufficient for $X$ to have no quotient space isomorphic to $l_{1}$, and if $\left(p_{k}\right)$ is unconditional, then it is also necessary that $\left(p_{h}\right)$ is shrinking.

$\mathrm{T}$ h e or em 4. Suppose that $\operatorname{Im} p_{k}, k=1,2, \ldots$, has no quotient space isomorphic to $c_{0}$. It is sufficient for $X$ to have no quotient space isomorphic to $c_{0}$, and if $\left(p_{k}\right)$ is unconditional, then it is also necessary that both $\left(p_{k}\right)$ and $\left(p_{k}^{*}\right)$ are shrinking.

These results are used to present a short proof for an example due to $\mathrm{S}$. F. Bellenot [12] and to deduce some results relating the linear-topological structures of a Banach space with an unconditional Schauder decomposition and its dual space. They also yield the following characterization of reflexivity.

Corolla ry 4. Suppose that $\left(p_{k}\right)$ is unconditional and $\operatorname{Im} p_{k}, k=1,2, \ldots$, is reflexive. Then, the following three assertions are equivalent:

$1^{\circ} X$ is reflexive,

$2^{\circ} X$ has no quotient space isomorphic to $c_{0}$,

$3^{\circ} X^{*}$ contains no subspace isomorphic to $l_{1}$. 